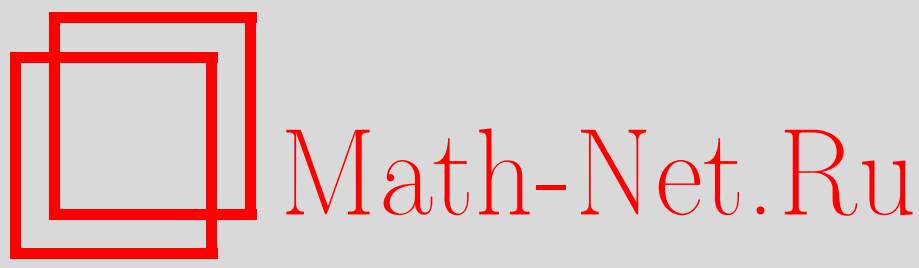

Ю. А. Неретин, Бета-интегралы и конечные ортогональные системы многочленов Вильсона, Матем. сб., 2002, том 193, номер 7, 131-148

DOI: https://doi.org/10.4213/sm670

Использование Общероссийского математического портала Math-Net.Ru подразумевает, что вы прочитали и согласны с пользовательским соглашением

http://www . mathnet.ru/rus/agreement

Параметры загрузки:

IP : 54.197 .130 .99

26 апреля 2023 г., 12:34:58 


\author{
Ю. А. Неретин
}

\title{
Бета-интегралы и конечные ортогональные системы многочленов Вильсона
}

В работе вьводится интеграл

$$
\frac{1}{2 \pi} \int_{-\infty}^{\infty}\left|\frac{\prod_{k=1}^{3} \Gamma\left(a_{k}+i s\right)}{\Gamma(2 i s) \Gamma(b+i s)}\right|^{2} d s=\frac{\Gamma\left(b-a_{1}-a_{2}-a_{3}\right) \prod_{1 \leqslant k<l \leqslant 3} \Gamma\left(a_{k}+a_{l}\right)}{\prod_{k=1}^{3} \Gamma\left(b-a_{k}\right)}
$$

и строится система ортогональных многочленов, весом для которых служит подынтегральное выражение. Вес убывает полиномиально, и поэтому лишп конечное число его моментов сходится. В итоге полученная система ортогоналњњых многочленов оказьвается конечной.

Также строится система ортогональных многочленов, связанная с ${ }_{5} \mathrm{H}_{5}$-форормулой Дуголла и интегралом Аски. Все три системы состоят из многочленов Вильсона вне области положительности обычного веса.

Библиографоия: 34 названия.

\section{§ 0. Введение}

В настоящей статье мы выводим бета-интеграл

$$
\frac{1}{2 \pi} \int_{-\infty}^{\infty}\left|\frac{\prod_{k=1}^{3} \Gamma\left(a_{k}+i s\right)}{\Gamma(2 i s) \Gamma(b+i s)}\right|^{2} d s=\frac{\Gamma\left(b-a_{1}-a_{2}-a_{3}\right) \prod_{1 \leqslant k<l \leqslant 3} \Gamma\left(a_{k}+a_{l}\right)}{\prod_{k=1}^{3} \Gamma\left(b-a_{k}\right)}
$$

(в литературе найти этот интеграл не удалось), строим систему ортогональных многочленов, связанных с этим интегралом, а также системы, связанные с интегралом Аски (0.5) и с ${ }_{5} H_{5}$-формулой Дуголла (0.6). Все полученные системы оказываются многочленами Вильсона (см. [1]-[3]) вне области положительности классического веса.

0.1. Бета-интегралы. Интеграл (0.1) является представителем большого семейства так называемых бета-интегралов. Мы упомянем лишь те из них, которые так или иначе появляются в данной статье: интеграл де Бранжа-Вильсона [1], [4] (см. также $[2 ; 3.6])$

$$
\frac{1}{2 \pi} \int_{-\infty}^{\infty}\left|\frac{\prod_{k=1}^{4} \Gamma\left(a_{k}+i s\right)}{\Gamma(2 i s)}\right|^{2} d s=\frac{\prod_{1 \leqslant k<l \leqslant 4} \Gamma\left(a_{k}+a_{l}\right)}{\Gamma\left(a_{1}+a_{2}+a_{3}+a_{4}\right)},
$$

вторая лемма Барнса (см. [2; теорема 2.4.3])

$$
\begin{aligned}
& \frac{1}{2 \pi} \int_{-\infty}^{\infty} \frac{\Gamma\left(a_{1}-i s\right) \Gamma\left(a_{2}-i s\right) \Gamma\left(b_{1}+i s\right) \Gamma\left(b_{2}+i s\right) \Gamma\left(b_{3}+i s\right)}{\Gamma\left(a_{1}+a_{2}+b_{1}+b_{2}+b_{3}+i s\right)} d s \\
& \quad=\frac{\prod_{k=1}^{3} \Gamma\left(a_{1}+b_{k}\right) \Gamma\left(a_{2}+b_{k}\right)}{\prod_{1 \leqslant k<l \leqslant 3} \Gamma\left(a_{1}+a_{2}+b_{k}+b_{l}\right)}
\end{aligned}
$$

Работа вьполнена при поддержке NWO (грант № 047-008-009). 
интеграл Нассраллаха-Рахмана (см. [5], [6])

$$
\frac{1}{2 \pi} \int_{-\infty}^{\infty}\left|\frac{\prod_{j=1}^{5} \Gamma\left(a_{j}+i s\right)}{\Gamma(2 i s) \Gamma\left(\sum_{1}^{5} a_{j}+i s\right)}\right|^{2} d s=2 \frac{\prod_{1 \leqslant k<l \leqslant 5} \Gamma\left(a_{k}+a_{l}\right)}{\prod_{k=1}^{5} \Gamma\left(a_{1}+a_{2}+a_{3}+a_{4}+a_{5}-a_{k}\right)}
$$

и интеграл Аски (см. [6])

$$
\frac{1}{2 \pi} \int_{-\infty}^{\infty} \frac{\Gamma(1-2 s) \Gamma(1+2 s)}{\prod_{k=1}^{4} \Gamma\left(a_{k}+s\right) \Gamma\left(a_{k}-s\right)} d s=\frac{\Gamma\left(a_{1}+a_{2}+a_{3}+a_{4}-3\right)}{\prod_{1 \leqslant k<l \leqslant 4} \Gamma\left(a_{k}+a_{l}-1\right)} .
$$

В последнем случае подынтегральная функция имеет полюсы $s= \pm n / 2, n=$ $1,2, \ldots$, на контуре интегрирования.

Интеграл можно понимать двумя способами.

Первый способ. Фиксируем $\alpha$ такое, что $0<\alpha<1$. Далее рассмотрим интеграл от $-M-\alpha$ до $N+\alpha$, где $M, N$ - целые положительные числа. Мы будем понимать этот интеграл как интеграл в смысле главного значения вблизи каждого полюса. При $\operatorname{Re} \sum a_{j}<3$ существует предел этого интеграла при $M, N \rightarrow+\infty$.

Другой способ - взять интеграл от $-M-\alpha$ до $N+\alpha$ по контуру, обходяшему полюсы сверху, и перейти к пределу при $M, N \rightarrow+\infty$. Оба варианта совпадут ввиду четности подынтегральной функции (поправки в парах полюсов $\pm k / 2$ сокрашаются).

Более полные списки бета-интегралов содержатся в [5], [6], есть также многочисленные $q$-аналоги (см. [5]-[7]), дискретные аналоги и многомерные (см. [8], [9]) аналоги бета-интегралов. Из дискретных аналогов нам понадобится следующая формула Дуголла

$$
\sum_{n=-\infty}^{\infty} \frac{\alpha+n}{\prod_{j=1}^{4} \Gamma\left(a_{j}+\alpha+n\right) \Gamma\left(a_{j}-\alpha-n\right)}=\frac{\sin 2 \pi \alpha}{2 \pi} \frac{\Gamma\left(a_{1}+a_{2}+a_{3}+a_{4}-3\right)}{\prod_{1 \leqslant j<k \leqslant 4} \Gamma\left(a_{j}+a_{k}-1\right)}
$$

ЗАмечАниЕ. В формулах (0.1)-(0.4) мы предполагаем, чтоо параметры $a_{j}, b_{j}$ вешественны и положительны. Если, например, мы перепишем равенство (0.2) в виде

$$
\frac{1}{2 \pi} \int_{-\infty}^{\infty} \frac{\prod_{k=1}^{4} \Gamma\left(a_{k}+i s\right) \Gamma\left(a_{k}-i s\right)}{\Gamma(2 i s) \Gamma(-2 i s)} d s=\frac{\prod_{1 \leqslant k<l \leqslant 4} \Gamma\left(a_{k}+a_{l}\right)}{\Gamma\left(a_{1}+a_{2}+a_{3}+a_{4}\right)},
$$

то (в силу аналитического продолжения) полученное равенство будет верно при $\operatorname{Re} a_{j}>0$. Это ограничение можно заменить на более слабое $a_{k}+a_{l} \neq 0,-1,-2, \ldots$ (для всех пар $k, l)$, но тогда придется изменить контур интегрирования (см. п. 0.4) или добавить сумму вычетов в правой части. Так же можно понимать и остальњые интегралы (0.1)-(0.4) и (2.2)-(2.5).

0.2. Интеграл Нассраллаха-Рахмана. Наш вывод интеграла (0.1) чрезвычайно прост, но сам этот интеграл может быть получен путем вырождений и гипергеометрических преобразований из следующего замечательного интеграла 
Насраллаха-Рахмана

$$
\begin{aligned}
& \int_{-\infty}^{\infty}\left|\frac{\Gamma(a+i s) \Gamma(b+i s) \Gamma(c+i s) \Gamma(d+i s) \Gamma(f+i s) \Gamma(g+i s)}{\Gamma(2 i s) \Gamma(\lambda+i s) \Gamma(\mu+i s)}\right|^{2} d s \\
& =\frac{4 \pi^{3} \sin (a+b+c+d) \pi \Gamma(a+b) \Gamma(a+c) \Gamma(a+d) \Gamma(1+2 a)}{\sin (b+c) \pi \sin (b+d) \pi \sin (c+d) \pi} \\
& \quad \times \frac{\Gamma(a+f) \Gamma(f-a) \Gamma(g+a) \Gamma(g-a)}{\Gamma(1+a-b) \Gamma(1+a-c) \Gamma(1+a-d) \Gamma(\lambda+a) \Gamma(\lambda-a) \Gamma(\mu+a) \Gamma(\mu-a)} \\
& \quad \times{ }_{9} F_{8}\left[\begin{array}{l}
2 a, 1+a, a+b, a+c, a+d, a+f, a+g, 1+a-\lambda, 1+a-\mu \\
a, 1+a-b, 1+a-c, 1+a-d, 1+a-f, 1+a-g, a+\lambda, a+\mu
\end{array}\right] \\
& \quad+\cdots,
\end{aligned}
$$

где многоточие означает сумму трех аналогичных слагаемых, получаемых при последовательной замене $a$ на $b, c$ и $d$.

Мы обсуждаем несколько интегралов (2.2)-(2.5), промежуточных между интегралами (0.2) и (0.1) и интегралом Насраллаха-Рахмана. Все они могут быть получены из (0.7) вырождением и гипергеометрическими преобразованиями (я однако нигде не встречал окончательных формул (2.2)-(2.5); они заслуживают того, чтобы быть выписанньпи (в особенности интегральное представление (2.2) для $\left.{ }_{3} F_{2}(1)\right)$, к тому же наши вычисления очень просты и идейно, и технически).

0.3. Конечные системы ортогональных многочленов. Мы явно выписываем систему многочленов $p\left(s^{2}\right)$, ортогональных относительно веса

$$
w_{1}(s)=\left|\frac{\prod_{k=1}^{3} \Gamma\left(a_{k}+i s\right)}{\Gamma(2 i s) \Gamma(b+i s)}\right|^{2} .
$$

Заметим, что этот вес убывает как $s^{-2\left(b-a_{1}-a_{2}-a_{3}\right)-1}$, поэтому лишш конечное число моментов $\int_{0}^{\infty} s^{2 k} w_{1}(s) d s$ имеет смысл. Поэтому наша система ортогональных многочленов конечна по определению.

Мы также строим систему ортогональных многочленов по дискретному весу

$$
w_{2}(s)=\sum c_{n} \delta_{n}
$$

где $\delta_{n}$ - дельта-функция, сосредоточенная в точке $n$, а $c_{n}$ - слагаемые формулы Дуголла (0.6).

Этот вес тоже полиномиально убывает. Он иногда положителен, иногда знаконеопределенен, в зависимости от $a_{j}$ и $\alpha$. Последнее, с точки зрения игры в формулы, не имеет большого значения.

Наконец мы строим (опять-таки конечную) систему многочленов, которая соответствует весу $w_{3}(s)$, где $w_{3}(s)$ - подынтегральное выражение в интеграле Аски (0.5). Этот вес не является положительным, более того, он не является мерой, однако интегрировать по нему можно.

Все три полученные системы оказываются многочленами Вильсона. Причем системы многочленов, связанных с весами $w_{2}(s)$ и $w_{3}(s)$, совпадают (с точностью до поправочного множителя в соотношениях ортогональности).

Конечные системы ортогональных многочленов (с непрерывньг весом) были впервые обнаружены Романовским [10] в 1929 (он построил аналоги многочленов 
Якоби). Аски в [11] получил систему многочленов, связанных с бета-интегралом Рамануджана. Различные конечные семейства ортогональных многочленов рассматривались в серии статей Лески [12]-[15], некоторые приложения таких конструкций можно найти в [16], [17].

0.4. Обозначения. В тексте используются следующие обозначения: $(a)_{k}:=$ $a(a+1) \cdots(a+k-1)-$ символ Похгаммера,

$$
{ }_{q} F_{p}\left[\begin{array}{c}
a_{1}, \ldots, a_{q} \\
b_{1}, \ldots, b_{p}
\end{array} ; z\right]={ }_{q} F_{p}\left(a_{1}, \ldots, a_{q} ; b_{1}, \ldots, b_{p} ; z\right):=\sum_{k=0}^{\infty} \frac{\left(a_{1}\right)_{k} \cdots\left(a_{q}\right)_{k}}{k !\left(b_{1}\right)_{k} \cdots\left(b_{p}\right)_{k}} z^{k}
$$

- обобщенная гипергеометрическая функция,

$$
\Gamma\left[\begin{array}{c}
u_{1}, \ldots, u_{l} \\
v_{1}, \ldots, v_{m}
\end{array}\right]:=\frac{\Gamma\left(u_{1}\right) \cdots \Gamma\left(u_{l}\right)}{\Gamma\left(v_{1}\right) \cdots \Gamma\left(v_{m}\right)}
$$

интегралы Меллина-Барнса

$$
\frac{1}{2 \pi i} \int_{-i \infty}^{i \infty} \Gamma\left[\begin{array}{c}
a_{1}+s, \ldots, a_{m}+s, b_{1}-s, \ldots, b_{n}-s \\
c_{1}-s, \ldots, c_{k}-s, d_{1}+s, \ldots, d_{l}+s
\end{array}\right] z^{-s} d s
$$

Предполагается, что интегрирование ведется по контуру, ведущему из $-i \infty$ в $+i \infty$, разделяющему левую серию полюсов $s=-a_{1}-j, \ldots,-a_{m}-j$, где $j=0,1,2, \ldots, \infty$, и правую серию полюсов $s=b_{1}+j, \ldots, s=b_{n}+j$. Мы полагаем, что ни один полюс из левых серий не совпадает ни с одним полюсом из правых серий (и, в частности, желаемый контур существует). Интеграл Меллина-Барнса по стандартньп правилам записывается в виде конечной суммы гипергеометрических функций с Г-множителями. Правила для выписывания при $m+n \geqslant k+l$ (у нас это всегда выполнено) можно найти в книгах Слейтер [18] и Маричева [19], см. также [20].

Частично работа была выполнена в Институте математических исследований (Беркли) и Институте им. Э. Шрёдингера (Вена). Автор выражает благодарность администрации этих институтов за гостеприимство.

\section{§ 1. Предварительные сведения и предварительные вычисления}

1.1. Идексное гипергеометрическое преобразование. Фиксируем $a, b>0$. Пусть $f$ - функция на полупрямой $x \geqslant 0$. Идексное гипергеометрическое преобразование $J_{a, b}$ задается формулой

$$
J_{a, b} f(s)=\frac{1}{\Gamma(a+b)} \int_{0}^{\infty} f(x)_{2} F_{1}(a+i s, a-i s ; a+b ;-x) x^{a+b-1}(1+x)^{a-b} d x .
$$

Обратное преобразование имеет вид

$$
J_{a, b}^{-1} f(x)=\frac{1}{\pi \Gamma(a+b)} \int_{0}^{\infty} f(s)_{2} F_{1}(a+i s, a-i s ; a+b ;-x)\left|\frac{\Gamma(a+i s) \Gamma(b+i s)}{\Gamma(2 i s)}\right|^{2} d s .
$$

Преобразование $J$ является унитарным преобразованием

$$
L^{2}\left(\mathbb{R}_{+}, x^{a+b-1}(1+x)^{a-b}\right) \rightarrow L^{2}\left(\mathbb{R}_{+},\left|\frac{\Gamma(a+i s) \Gamma(b+i s)}{\Gamma(2 i s)}\right|^{2}\right)
$$


Условие унитарности (формула Планшереля) в явном виде имеет вид

$$
\int_{0}^{\infty} f_{1}(x) \overline{f_{2}(x)} x^{a+b-1}(1+x)^{a-b} d x=\frac{1}{\pi} \int_{0}^{\infty} g_{1}(s) \overline{g_{2}(s)}\left|\frac{\Gamma(a+i s) \Gamma(b+i s)}{\Gamma(2 i s)}\right|^{2} d s .
$$

О свойствах этого преобразования см. [21]-[26]. Преобразование $J$ называется также преобразованием Олевского, преобразованием Якоби, преобразованием Фурье-Якоби и обобщенным преобразованием Фурье, обнаружено оно Германом Вейлем в 1910 году.

1.2. Гипергеометрическая функция Гаусса. Мы используем несколько простых фактов о гипергеометрических функциях ${ }_{2} F_{1}$ : в частности, формулу Гаусса (см. [2; теорема 2.2,2], [27; (2.1.14)])

$$
{ }_{2} F_{1}(a, b ; c ; 1)=\frac{\Gamma(c) \Gamma(c-a-b)}{\Gamma(c-a) \Gamma(c-b)}, \quad \operatorname{Re}(c-a-b)>0,
$$

и формулу Больиа (см. [27; (2.1.22-23)])

$$
\begin{aligned}
{ }_{2} F_{1}(a, b ; c ;-x) & =(1+x)^{-a}{ }_{2} F_{1}\left(a, c-b ; c ; \frac{x}{x+1}\right) \\
& =(1+x)^{-b}{ }_{2} F_{1}\left(c-a, b ; c ; \frac{x}{x+1}\right) \\
& =(1+x)^{c-a-b}{ }_{2} F_{1}(c-a, c-b ; c ;-x) .
\end{aligned}
$$

Напомним, что функция ${ }_{2} F_{1}(a, b ; c ; x)$ аналитически продолжается на полупрямую $x<0$. Асиптотика функции ${ }_{2} F_{1}(a+i s, a-i s ; a+b ;-x)$ при $x \rightarrow+\infty$ имеет вид

$$
{ }_{2} F_{1}(a+i s, a-i s ; a+b ;-x)=\psi(x) x^{-a},
$$

где $\psi$ - ограниченная функция при $s \neq 0$ и $\psi(x) / \ln (x)$ - ограниченная функция при $s=0$ (более точное выражение см. в $[27 ;(2.3 .2 .9)])$.

Асимптотика функции ${ }_{2} F_{1}(a+i s, a-i s ; a+b ;-x)$ при $s \rightarrow+\infty$ имеет вид

$$
{ }_{2} F_{1}(a+i s, a-i s ; a+b ;-x)=\lambda(s) s^{-a-b+1 / 2},
$$

где $\lambda(s)$ - ограниченная функция (см. формулу Ватсона в $[27 ;(2.3 .2 .17)])$.

Напомним три представления Гауссовой гипергеометрической функции в виде Барнсовских интегралов (везде $x>0$ ), (см. [27, (2.1.15)], [19; $§ 2.10,11(1), 19(1)$, $22(1)],[2$; теоремы $2.4 .1,2.4 .2])$ :

$$
\begin{gathered}
\frac{1}{2 \pi i} \frac{\Gamma(c)}{\Gamma(a) \Gamma(b)} \int_{-i \infty}^{i \infty} \frac{\Gamma(a-s) \Gamma(b-s) \Gamma(s)}{\Gamma(c-s)} x^{-s} d s={ }_{2} F_{1}(a, b ; c ;-x) \\
\frac{1}{2 \pi i} \frac{\Gamma(c)}{\Gamma(a) \Gamma(b) \Gamma(c-a) \Gamma(c-b)} \int_{-i \infty}^{i \infty} \Gamma(s) \Gamma(s+c-a-b) \Gamma(a-s) \Gamma(b-s) x^{-s} d s \\
={ }_{2} F_{1}(a, b ; c ; 1-x) \\
\frac{1}{2 \pi i} \Gamma(c) \int_{-i \infty}^{i \infty} \frac{\Gamma(s) \Gamma(s+c-a-b)}{\Gamma(s+c-a) \Gamma(s+c-b)} x^{-s} d s \\
= \begin{cases}(1-x)^{c-1}{ }_{2} F_{1}(a, b ; c ; 1-x), & x>1 \\
0, & x<1\end{cases}
\end{gathered}
$$


1.3. Г-функция. Мы будем использовать следующее интегральное представление бета-функции

$$
\int_{0}^{\infty} \frac{x^{\alpha-1} d x}{(1+x)^{\rho}}=B(\alpha, \rho-\alpha)
$$

заменой переменной $x /(x+1)=z$ этот интеграл приводится к обычному определению бета-функции.

Асиптотика функции $\Gamma(z)$ задается формулой Стирлинга

$$
\Gamma(z)=\sqrt{2 \pi} z^{z-1 / 2} e^{-z}\left(1+O\left(\frac{1}{z}\right)\right), \quad z \rightarrow \infty, \quad \arg z<\pi-\varepsilon .
$$

В частности (см. [27; $(1.18 .3),(1.18 .6)])$,

$$
\begin{gathered}
\frac{\Gamma(z+a)}{\Gamma(z+b)} \sim z^{a-b}, \quad z \rightarrow \infty, \quad \arg z<\pi-\varepsilon, \\
|\Gamma(a+i s)| \sim \sqrt{2 \pi}|s|^{a-1 / 2} e^{-|s| \pi / 2}, \quad s \rightarrow+\infty .
\end{gathered}
$$

1.4. Преобразование Меллина. Рассмотрим функцию $f$ на полупрямой $x \geqslant 0$. Ее преобразование Меллина задается формулой

$$
f^{*}(s)=\int_{0}^{\infty} x^{s} f(x) \frac{d x}{x} .
$$

Формула обращения имеет вид

$$
f(x)=\frac{1}{2 \pi i} \int_{-i \infty}^{i \infty} f^{*}(s) x^{-s} d s .
$$

Преобразование Меллина переводит свертку

$$
\int_{0}^{\infty} f_{1}\left(\frac{x}{t}\right) f_{2}(t) \frac{d t}{t}
$$

в произведение преобразований Меллина $f_{1}^{*}(s) f_{2}^{*}(s)$. По поводу точных формулировок см., например, [19].

Функция $f(a x)$ (при $a>0)$, очевидно, переходит в $a^{-s} f^{*}(s)$, а функция $x^{\alpha} f(x)$ - в $f^{*}(s+\alpha)$.

1.5. Нам понадобятся некоторые интегралы вида

$$
\begin{aligned}
\int_{0}^{\infty} & \frac{x^{\alpha-1}}{(x+z)^{\rho}}{ }^{2} F_{1}(p, q ; r ;-x) d x \\
\quad= & \frac{1}{2 \pi i} z^{\alpha-\rho} \Gamma\left[\begin{array}{c}
r \\
p, q, \rho
\end{array}\right] \int_{-i \infty}^{i \infty} \Gamma\left[\begin{array}{c}
s+\alpha, \rho-s-\alpha, p+s, q+s,-s \\
r+s
\end{array}\right] z^{s} d s .
\end{aligned}
$$

Интеграл Меллина-Барнса в правой части выражается как линейная комбинация двух ${ }_{3} F_{2}$.

Мы приведем в качестве образца (вполне стандартное, см. [19]) доказательство тождества (1.10), тоже вполне стандартного (см. [20; (2.21.1.16)]).

Для этого представим $x^{\alpha}(x+z)^{-\rho}$ в виде обратного преобразования Меллина

$$
x^{\alpha}(x+z)^{-\rho}=\frac{z^{\alpha-\rho}}{2 \pi \Gamma(\rho)} \int_{-i \infty}^{i \infty} \Gamma(s+\alpha) \Gamma(\rho-s-\alpha) z^{s} x^{-s} d s
$$

(чтобы убедиться в этом, достаточно вычислить прямое преобразование Меллина). Применяя интеграл Барнса (1.7) и формулу свертки для преобразования Меллина, получаем (1.10). 
1.6. Следствия из формулы (1.10).

Лемма 1.1. а) Оператор $J$ переводит функцию

$$
(1+x)^{-a-c} \quad \text { в } \frac{\Gamma(c+i s) \Gamma(c-i s)}{\Gamma(c+a) \Gamma(c+b)} .
$$

b) Оператор $J$ переводит функиию

$$
\frac{(1+x)^{b-a}}{(x+z)^{c+b}} \quad \text { в } \quad \frac{\Gamma(c+i s) \Gamma(c-i s)}{\Gamma(c+a) \Gamma(c+b)}{ }_{2} F_{1}\left[\begin{array}{c}
c+i s, c-i s \\
c+a
\end{array} ; 1-z\right] .
$$

с) Oператор $J$ переводит функиию

$$
x^{-u-a} \quad \text { в } \frac{\Gamma(-u+b)}{\Gamma(a+u)} \cdot \frac{\Gamma(u+i s) \Gamma(u-i s)}{\Gamma(b+i s) \Gamma(b-i s)} .
$$

ДокАЗАТЕЛЬСТво. Нужно подставить соответствующие $f$ в формулу (1.1). Мы можем просто сослаться на (2.21.1.4), (2.21.1.5), (2.21.1.15) Прудникова, Брычкова, Маричева [20], или на [28; 7.512].

Лучше, однако, объяснить, что происходит и почему при одних значениях параметров интеграл (1.10) вычисляется, а при других - нет.

1) При $\alpha=r$ в правой части (1.10) под интегралом два Г-множителя сокращаются. Остается интеграл Барнса типа (1.8), что доказывает утверждение b).

2) Утверждение a) получается подстановкой $z=1$ в утверждение $\mathrm{b})$.

3) Подставим $z=1$ в (1.10). При $r=p+q+\rho$ правая часть вычисляется по второй лемме Барнса (0.3). Это дает с).

1.7. Интегралы с произведениями гипергеометрических функций. Прямое применение формулы свертки для преобразования Меллина дает

$$
\begin{aligned}
\int_{0}^{\infty} & x^{\alpha-1}{ }_{2} F_{1}\left[\begin{array}{cc}
p, q \\
r
\end{array}-\omega x\right]{ }_{2} F_{1}\left[\begin{array}{cc}
u, v \\
w
\end{array}-\widetilde{\omega} x\right] d x \\
= & \frac{1}{2 \pi i} \omega^{-\alpha} \Gamma\left[\begin{array}{c}
r, w \\
u, v, p, q
\end{array}\right] \\
& \times \int_{-i \infty}^{i \infty} \Gamma\left[\begin{array}{c}
\alpha+s, u+s, v+s, p-\alpha-s, q-\alpha-s,-s \\
r-\alpha-s, w+s
\end{array}\right]\left(\frac{\omega}{\widetilde{\omega}}\right)^{-s} d s .
\end{aligned}
$$

Если в это тождество подставить $\alpha=w=r$, то четыре Г-множителя под интегралом в правой части сокрашаются. Применяя формулу Барнса (1.8), получаем

$$
\begin{aligned}
\int_{0}^{\infty} x_{2}^{r-1}{ }_{2} F_{1}\left[\begin{array}{cr}
p, q \\
r
\end{array}-\omega x\right]{ }_{2} F_{1}\left[\begin{array}{cc}
u, v \\
r
\end{array} ;-\widetilde{\omega} x\right] d x \\
=\omega^{-r+u} \widetilde{\omega}^{-u} \Gamma\left[\begin{array}{c}
r, r, p-r+u, q-r+u, p-r+v, q-r+v \\
u, v, p, q, p+q+u+v-2 r
\end{array}\right] \\
\quad \times{ }_{2} F_{1}\left[\begin{array}{c}
p-r+u, q-r+u \\
p+q+u+v-2 r
\end{array} ; 1-\frac{\omega}{\omega}\right] .
\end{aligned}
$$

Это формула (2.21.9.7) таблиц [20] (там отсутствует штрих во втором индексе гипергеометрической функции в правой части). 
Нам также понадобится интеграл

$$
\begin{aligned}
& \int_{0}^{1} z^{\mu-1}(1-z)^{\nu-1}{ }_{2} F_{1}\left[\begin{array}{c}
\alpha, \beta \\
\nu
\end{array} ; 1-z\right]{ }_{2} F_{1}\left[\begin{array}{c}
\varphi, \psi \\
\xi
\end{array} 11-z\right] d z \\
& \quad=\frac{1}{2 \pi i} \Gamma\left[\begin{array}{c}
\nu, \xi \\
\varphi, \psi, \xi-\varphi, \xi-\psi
\end{array}\right] \\
& \quad \times \int_{-i \infty}^{i \infty} \Gamma\left[\begin{array}{c}
\mu+s, \mu+\nu-\alpha-\beta+s, \varphi+s, \psi+s,-s, \xi-\varphi-\psi+s \\
\nu+\mu-\alpha+s, \mu+\nu-\beta+s
\end{array}\right] d s .
\end{aligned}
$$

Для вывода этой формулы запишем $(1-z)^{\nu-1}{ }_{2} F_{1}(\alpha, \beta ; \nu ; 1-z)$ как интеграл (1.9), а функцию ${ }_{2} F_{1}(\varphi, \psi ; \xi ; 1-z)$ как интеграл (1.8). Далее применяем формулу свертки.

\section{9. Следствия из формулы (1.11).}

ЛЕмма 1.2. а) Преобразование J переводит

$$
{ }_{2} F_{1}\left[\begin{array}{c}
p+b, q+b \\
a+b
\end{array} ;-\frac{x}{y}\right](1+x)^{b-a}
$$

$B$

$$
\begin{gathered}
\frac{y^{b-q} \Gamma(a+b)}{\Gamma(p+q) \Gamma(p+b) \Gamma(q+b)} \cdot \frac{\Gamma(p+i s) \Gamma(p-i s) \Gamma(q+i s) \Gamma(q-i s)}{\Gamma(a+i s) \Gamma(a-i s)} \\
\times{ }_{2} F_{1}\left[\begin{array}{c}
p+i s, p-i s \\
p+q
\end{array} ; 1-y\right] .
\end{gathered}
$$

b) Преобразование $J$ переводит

$$
{ }_{2} F_{1}\left[\begin{array}{c}
p+b, q+b \\
a+b
\end{array} ;-x\right](1+x)^{b-a}
$$

B

$$
\frac{\Gamma(a+b)}{\Gamma(p+q) \Gamma(p+b) \Gamma(q+b)} \cdot \frac{\Gamma(p+i s) \Gamma(p-i s) \Gamma(q+i s) \Gamma(q-i s)}{\Gamma(a+i s) \Gamma(a-i s)} .
$$

с) Преобразование $J$ переводит

$$
{ }_{2} F_{1}\left[\begin{array}{c}
a+c, a+d \\
a+b+c+d
\end{array} ;-x\right]
$$

B

$$
\frac{\Gamma(a+b+c+d) \cdot \Gamma(c+i s) \Gamma(c-i s) \Gamma(d+i s) \Gamma(d-i s)}{\Gamma(a+c) \Gamma(a+d) \Gamma(b+c) \Gamma(b+d) \Gamma(c+d)}
$$


ДокАЗАТЕльство. Утверждение а) получается непосредственным применением формулы (1.12). Утверждение b) получается из а) подстановкой $y=1$ (разумеется, лучше сразу подставить $\omega=\widetilde{\omega}$ в (1.11)).

Выведем с). Подставляя $f={ }_{2} F_{1}[. .$.$] в (1.11) и применяя преобразование Боль-$ ца, получаем

$$
\frac{1}{\Gamma(a+b)} \int_{0}^{\infty} x^{a+b-1}{ }_{2} F_{1}\left[\begin{array}{c}
b+d, b+c \\
a+b+c+d
\end{array} ;-x\right]{ }_{2} F_{1}\left[\begin{array}{c}
a+i s, a-i s \\
a+b
\end{array} ;-x\right] d x .
$$

Далее в правой части (1.12) под интегралом два Г-множителя сокращаются, и мы получаем

$$
\begin{aligned}
& \frac{1}{2 \pi i} \Gamma\left[\begin{array}{c}
a+b+c+d \\
a+i s, a-i s, b+c, b+d
\end{array}\right] \\
& \quad \times \int_{-i \infty}^{i \infty} \Gamma\left[\begin{array}{c}
a+i s+t, a-i s+t, d-a-t, c-a-t,-t] \\
c+d-t
\end{array}\right] d t
\end{aligned}
$$

Остается применить вторую лемму Барнса (0.3).

\section{§2. Вырожденные случаи интеграла Насраллаха-Рахмана}

В этом параграфе мы применяем формулу Планшереля (1.3) для индексного преобразования к различным парам функций из лемм 1.1-1.2.

Параметры $a, b, c, d, e, f, u, v$ для краткости формул предполагаются вешественными положительньми.

2.1. Интеграл де Бранжа-Вильсона. Применим формулу Планшереля (1.3) к паре функций $(1+x)^{-a-c}$ и $(1+x)^{-a-d}$. В левой части имеем бета-интеграл

$$
\int_{0}^{\infty} \frac{x^{a+b-1} d x}{(1+x)^{a+b+c+d}}
$$

а в правой части (в силу леммы 1.1.а)) - интеграл де Бранжа-Вильсона (с точностью до Г-множителей). Вычисляя (2.1), получаем (0.2).

Этот вывод интеграла де Бранжа-Вильсона известен (см. [23]).

2.2. Вывод бета-интеграла (0.1). Достаточно применить формулу Планшереля к паре функций $x^{-u-a}$ и $x^{-v-a}$ (см. лемму 1.1.c)).

2.3. Применим формулу Планшереля (1.3) к паре функций

$$
(1+x)^{-a-e} \quad \text { и } \quad{ }_{2} F_{1}\left[\begin{array}{c}
a+c, a+d \\
a+b+c+d
\end{array} ;-x\right] .
$$

В левой части имеем

$$
\int_{0}^{\infty} x^{a+b-1}(1+x)_{2}^{-b-e} F_{1}\left[\begin{array}{c}
a+c, a+d \\
a+b+c+d
\end{array} ;-x\right] d x .
$$

Преобразуя это по формуле Больца, получаем

$$
\int_{0}^{1} z^{a+b-1}(1-z)^{c+e-1} F_{1}\left[\begin{array}{c}
a+c, b+c \\
a+b+c+d
\end{array} ; z\right] d z
$$


Это стандартное интегральное представление для ${ }_{3} F_{2}($ см. $[2 ;(2.2 .4)])$, и, окончательно, левая часть равна

$$
\frac{\Gamma(a+b) \Gamma(c+e)}{\Gamma(a+b+c+e)}{ }_{3} F_{2}\left[\begin{array}{c}
a+c, b+c, a+b \\
a+b+c+d, a+b+c+e
\end{array} ; 1\right]
$$

Приравнивая правую часть формулы Планшереля к левой, получаем

$$
\begin{aligned}
\frac{1}{\pi} \int_{0}^{\infty} & \left|\frac{\Gamma(a+i s) \Gamma(b+i s) \Gamma(c+i s) \Gamma(d+i s) \Gamma(e+i s)}{\Gamma(2 i s)}\right|^{2} d s \\
= & \frac{\Gamma(a+b) \Gamma(a+c) \Gamma(a+d) \Gamma(a+e) \Gamma(b+c) \Gamma(b+d) \Gamma(b+e) \Gamma(c+d) \Gamma(c+e)}{\Gamma(a+b+c+d) \Gamma(a+b+c+e)} \\
& \times{ }_{3} F_{2}\left[\begin{array}{c}
a+c, b+c, a+b \\
a+b+c+d, a+b+c+e
\end{array}\right]
\end{aligned}
$$

среди Г-множителей в числителе отсутствует $\Gamma(e+d)$.

ЗАмечАниЕ. Очевидно, левая часть равенства симметрична по $a, b, c, d, e$. Симметричность правой части равносильна формуле Куммера (см. [2; следствие 3.3.5]).

2.4. Теперь применим форомулу Планшереля (1.3) к паре функций

$$
{ }_{2} F_{1}\left[\begin{array}{c}
a+c, a+d \\
a+b+c+d
\end{array} ;-x\right] \text { и }{ }_{2} F_{1}\left[\begin{array}{c}
a+e, a+f \\
a+b+e+f
\end{array} ;-x\right]
$$

(см. лемму 1.2.c)). В левой части имеем

$$
\begin{gathered}
\int_{0}^{\infty} x^{a+b-1}{ }_{2} F_{1}\left[\begin{array}{c}
a+c, a+d \\
a+b+c+d
\end{array} ;-x\right]{ }_{2} F_{1}\left[\begin{array}{c}
a+e, a+f \\
a+b+e+f
\end{array} ;-x\right](1+x)^{a-b} d x \\
=\int_{0}^{\infty} x^{a+b-1}{ }_{2} F_{1}\left[\begin{array}{c}
b+d, b+c \\
a+b+c+d
\end{array} ;-x\right]{ }_{2} F_{1}\left[\begin{array}{c}
a+e, a+f \\
a+b+e+f
\end{array} ;-x\right] d x .
\end{gathered}
$$

Применяя формулу (1.11), получаем

$$
\begin{aligned}
\frac{1}{\pi} \int_{0}^{\infty} & \left|\frac{\Gamma(a+i s) \Gamma(b+i s) \Gamma(c+i s) \Gamma(d+i s) \Gamma(e+i s) \Gamma(f+i s)}{\Gamma(2 i s)}\right|^{2} d s \\
= & \frac{1}{2 \pi i} \Gamma(a+c) \Gamma(a+d) \Gamma(c+d) \Gamma(b+e) \Gamma(b+f) \Gamma(e+f) \\
& \times \int_{-i \infty}^{i \infty} \Gamma\left[\begin{array}{c}
a+b+s, a+e+s, a+f+s, d-a-s, c-a-s,-s \\
c+d-s, a+b+e+f+s
\end{array}\right] d s .
\end{aligned}
$$

Выражение в правой части расписьвается как линейная комбинация трех функций ${ }_{4} F_{3}(1)$. 
2.5. Теперь мы применим формулу Планшереля к паре функций

$$
{ }_{2} F_{1}\left[\begin{array}{c}
p+b, q+b \\
a+b
\end{array} ;-x\right](1+x)^{b-a} \text { и }{ }_{2} F_{1}\left[\begin{array}{c}
u+b, v+b \\
a+b
\end{array} ;-x\right](1+x)^{b-a}
$$

(их индексные преобразования вычислены в лемме 1.2.b)).

Мы должны вычислить интеграл

$$
\int_{0}^{\infty} x^{a+b-1}(1+x)^{b-a}{ }_{2} F_{1}\left[\begin{array}{c}
p+b, q+b \\
a+b
\end{array} ;-x\right]{ }_{2} F_{1}\left[\begin{array}{c}
u+b, v+b \\
a+b
\end{array} ;-x\right] d x .
$$

Применяя формулу Больца, приводим его к виду

$$
\begin{aligned}
& \int_{0}^{1} y^{a+b-1}(1-y)^{u+p_{2}} F_{1}\left[\begin{array}{c}
p+b, a-q \\
a+b
\end{array} ; y\right]{ }_{2} F_{1}\left[\begin{array}{c}
u+b, a-v \\
a+b
\end{array} ; y\right] d y \\
& =\int_{0}^{1}(1-z)^{a+b-1} z^{u+p}{ }_{2} F_{1}\left[\begin{array}{c}
p+b, a-q \\
a+b
\end{array} ; 1-z\right]{ }_{2} F_{1}\left[\begin{array}{c}
u+b, a-v \\
a+b
\end{array} ; 1-z\right] d z .
\end{aligned}
$$

Остается применить формулу (1.13). В итоге получаем

$$
\begin{aligned}
\frac{1}{\pi} \int_{0}^{\infty} & \left|\frac{\Gamma(b+i s) \Gamma(p+i s) \Gamma(q+i s) \Gamma(u+i s) \Gamma(v+i s)}{\Gamma(2 i s) \Gamma(a+i s)}\right|^{2} d s \\
= & \frac{1}{2 \pi i} \Gamma\left[\begin{array}{c}
u+v, p+q, p+b, q+b \\
a-v, u-v
\end{array}\right] \\
& \times \int_{-i \infty}^{i \infty} \Gamma\left[\begin{array}{c}
u+p+s, u+q+s, b+u+s, a-v+s, v-u-s,-s \\
u+a+s, u+b+p+q+s
\end{array}\right] d s .
\end{aligned}
$$

ЗАмЕчАниЕ. Правая часть переписывается в виде

$$
\left.\begin{array}{rl}
\Gamma & {\left[\begin{array}{c}
u+v, p+q, p+b, q+b \\
a-v, u-v
\end{array}\right]} \\
& \times\left(\Gamma [ \begin{array} { c } 
{ v - u , u + p , u + q , u + b , a - v } \\
{ u + a , u + b + p + q }
\end{array} ] { } _ { 4 } F _ { 3 } \left[\begin{array}{c}
u+p, u+q, u+b, a-v \\
1+u-v, u+a, u+b+p+q
\end{array} ;\right.\right.
\end{array}\right] .
$$

У Рахмана [29] для интеграла (2.4) дается выражение вида ${ }_{7} F_{6}$ (см. также [30; (6.3.11)]). Оно, впрочем, легко приводится к форме ${ }_{4} F_{3}$ с помощью "nonterminating" ${ }_{7} F_{6}$-преобразования Уиппла, обсуждаемого у Бейли $[31 ; 4.4,6.3,7.5]$.

ЗАмечаниЕ. Левая часть тождества (2.4) симметрична по $b, p, q, u, v$. Для правой части очевидна лишш симметричность по $u, v$ и по $b, p, q$. Перестановка $b$ и $u$ местами ведет к 4 -членному ${ }_{4} F_{3}$-тождеству. Если мы подставим в него $a=$ $v-m$, где $m$ - целое неотрицательное, то два слагаемых занулятся (из-за $\Gamma(-m)$ в знаменателе) и мы получим ${ }_{4} F_{3}$-преобразование Уиппла (см. [2; теорема 3.3 .3$]$; оно упоминается ниже в п. 3.1).

2.6. Интеграл Насраллаха-Рахмана (0.4). Если $a=b+u+v+p+q$, то в правой части (2.4) два Г-множителя под интегралом сокращаются. Применяя вторую лемму Барнса (0.3), получаем (0.4) (по поводу формулы (0.7) см. [32], [33]). 
2.7. Умножая обе части равенства $(2.4)$ на $\Gamma^{-2}(b)$ и переходя к пределу при $b \rightarrow+\infty$, получаем

$$
\begin{aligned}
& \frac{1}{\pi} \int_{0}^{\infty}\left|\frac{\Gamma(p+i s) \Gamma(q+i s) \Gamma(u+i s) \Gamma(v+i s)}{\Gamma(2 i s) \Gamma(a+i s)}\right|^{2} d s=\frac{1}{2 \pi i} \Gamma\left[\begin{array}{c}
u+v, p+q \\
a-v, u-v
\end{array}\right] \\
& \quad \times \int_{-i \infty}^{i \infty} \Gamma\left[\begin{array}{c}
u+p+s, u+q+s, a-v+s, v-u-s,-s \\
u+a+s
\end{array}\right] d s
\end{aligned}
$$

(справа стоит линейная комбинация двух функций $\left.{ }_{3} F_{2}(1)\right)$.

\section{§ 3. Конечные системы ортогональных многочленов}

3.1. Многочлены Вильсона. В знаменитой работе Вильсона [1] были построены многочлены $p\left(s^{2}\right)$, ортогональные по весу

$$
w(s)=\frac{1}{\pi}\left|\frac{\Gamma(a+i s) \Gamma(b+i s) \Gamma(c+i s) \Gamma(d+i s)}{\Gamma(2 i s)}\right|^{2} .
$$

Они задаются формулой

$$
\begin{aligned}
& p_{n}\left(a, b, c, d ; s^{2}\right)=(a+b)_{n}(a+c)_{n}(a+d)_{n} \\
& \quad \times{ }_{4} F_{3}\left[\begin{array}{c}
-n, n+a+b+c+d-1, a+i s, a-i s \\
a+b, a+c, a+d
\end{array} \quad 1\right] .
\end{aligned}
$$

Соотношения ортогональности имеют вид

$$
\begin{aligned}
& \int_{0}^{\infty} p_{n}\left(a, b, c, d ; s^{2}\right) p_{m}\left(a, b, c, d ; s^{2}\right) w(s) d s \\
& =\frac{n ! \Gamma(a+b+n) \Gamma(a+c+n) \Gamma(a+d+n) \Gamma(b+c+n) \Gamma(b+d+n) \Gamma(c+d+n)}{\Gamma(a+b+c+d+n)(a+b+c+d+2 n-1)} \delta_{m, n} .
\end{aligned}
$$

Очевидно, многочлены $p_{n}\left(a, b, c, d ; s^{2}\right)$ симметричны по $b, c, d$. То, что они симметричны по всем четырем индексам $a, b, c, d$, равносильно ${ }_{4} F_{3}$-преобразованию Уиппла, упоминавшемуся выше в п. 2.5.

3.2. Доказательство ортогональности. Доказательств ортогональности многочленов Вильсона известно много, наша цель - дать образец рассуждения, которое дословно “проходит” в трех случаях “экзотической” ортогональности, обсуждаемых ниже.

Лемма 3.1. Пусть даны $N$-мерные линейнье пространства $V, W$ с базисами $e_{j}, f_{j}$. Зададим на $V \times W$ билинейную форму $\mathscr{B}(\cdot, \cdot)$ так, что

$$
\mathscr{B}\left(e_{k}, f_{l}\right)=\frac{\Gamma(\nu+k+l)}{\Gamma(\mu+\nu+k+l)},
$$

где $\mu, \nu$ фиксированы. Тогда система векторов

$$
R_{n}=\sum_{j=0}^{n} \frac{(-n)_{j}(n+\mu+\nu-1)_{j}}{(\nu)_{j} j !} e_{j}, \quad T_{n}=\sum_{j=0}^{n} \frac{(-n)_{j}(n+\mu+\nu-1)_{j}}{(\nu)_{j} j !} f_{j}
$$

биортогональна, т.е. $\mathscr{B}\left(R_{k}, T_{l}\right)=0$ при $k \neq l$. Кроме того,

$$
\mathscr{B}\left(T_{n}, R_{n}\right)=\frac{n ! \Gamma(\nu) \Gamma(\mu+n)}{\Gamma(\nu+n)) \Gamma(n+\mu+\nu-1)(\mu+\nu+2 n-1)} .
$$


ДокАЗАТЕЛЬСтво. Эта лемма просто имитирует соотношения ортогональности для многочленов Якоби. Берем в качестве $V=W$ пространство многочленов на отрезке $[0,1]$ со скалярным произведением

$$
\langle p, q\rangle=\frac{1}{\Gamma(\mu)} \int_{0}^{1} p(x) \overline{q(x)} x^{\nu-1}(1-x)^{\mu-1} d x .
$$

В качестве $e_{n}=f_{n}$ берем функции $x^{n}$. Тогда $\left\langle e_{k}, f_{l}\right\rangle$ задается формулой (3.3). Соответственно, $R_{n}=T_{n}$ - это многочлены Якоби $P^{\mu-1, \nu-1}(2 x-1)$ в стандартных обозначениях (см. [34; 10.8$])$.

Теперь возьмем в качестве $V=W$ пространство $L^{2}$ по весу Вильсона $w(s)$. Положим

$$
e_{k}(s)=\frac{(a+i s)_{k}(a-i s)_{k}}{\Gamma(a+c+k) \Gamma(a+d+k)}, \quad f_{k}(s)=\frac{(b+i s)_{k}(b-i s)_{k}}{\Gamma(b+c+k) \Gamma(b+d+k)} .
$$

Тогда в силу $(0.2)$

$$
\begin{aligned}
\int_{0}^{\infty} & e_{k}(s) f_{m}(s) w(s) d s \\
= & \frac{1}{\pi \Gamma(a+c+k) \Gamma(a+d+k) \Gamma(b+c+m) \Gamma(b+d+m)} \\
& \quad \times \int_{0}^{\infty}\left|\frac{\Gamma(a+k+i s) \Gamma(b+m+i s) \Gamma(c+i s) \Gamma(d+i s)}{\Gamma(2 i s)}\right|^{2} d s \\
= & \frac{\Gamma(c+d) \Gamma(a+b+k+m)}{\Gamma(a+b+c+d+k+m)} .
\end{aligned}
$$

Мы получили соотношения типа (3.3) для скалярных произведений. В обозначениях леммы 3.1 мы получаем

$$
R_{n}=\alpha_{n} p_{n}\left(a, b, c, d ; s^{2}\right), \quad T_{n}=\beta_{n} p_{n}\left(b, a, c, d ; s^{2}\right),
$$

где $\alpha_{n}, \beta_{n}$ - нормировочные множители, которые мы для краткости опускаем. Осталось вспомнить, что многочлены Вильсона симметричны по $a, b, c, d$. Поэтому $R_{n}$ и $T_{n}$ совпадают с точностью до множителя (собственно, это место и является удивительньп).

Формула (3.2) вытекает из (3.4).

3.3. Соотношения отогональности, связанные с интегралом (0.1). Рассмотрим вес

$$
w_{1}(s)=\frac{1}{\pi}\left|\frac{\Gamma(p+i s) \Gamma(u+i s) \Gamma(v+i s)}{\Gamma(2 i s) \Gamma(q+i s)}\right|^{2}
$$

на полупрямой $s \geqslant 0$.

Положим

$$
\begin{aligned}
e_{n}(s) & =\frac{\Gamma(-u-k+q)}{\Gamma(p+u+k)}(u+i s)_{k}(u-i s)_{k} \\
f_{n}(s) & =\frac{\Gamma(-v-k+q)}{\Gamma(p+v+k)}(v+i s)_{k}(v-i s)_{k}
\end{aligned}
$$


Тогда

$$
\begin{gathered}
\int_{0}^{\infty} e_{k}(s) f_{l}(s) w_{1}(s) d s=\frac{\Gamma(u+v+k+l) \Gamma(q-u-v-p-k-l)}{\Gamma(q-p)} \\
=\frac{(-1)^{k+l} \pi}{\Gamma(q-p) \sin \pi(q-u-v-p)} \frac{\Gamma(u+v+k+l)}{\Gamma(1-q+u+v+p+k+l)} .
\end{gathered}
$$

Мы видим, что скалярные произведения имеют вид (3.3). Далее наши рассуждения из п. 3.2 можно повторить дословно.

Есть, однако, путь еще более простой.

ЛЕмма 3.2. Пусть $\ell$ - линейный функционал на пространстве четных многочленов

причем для

$$
c_{0}+c_{1} s^{2}+\cdots+c_{N} s^{2 N}
$$

$$
h_{k}=(a+i s)_{k}(a-i s)_{k}
$$

выполнено

$$
\ell\left(h_{k}\right)=C \frac{\Gamma(a+b+k) \Gamma(a+c+k) \Gamma(a+d+k)}{\Gamma(a+b+c+d+k)} .
$$

Тогда при $k+l \leqslant N$ для многочленов Вильсона $p_{k}, p_{l}$ выполнено

$$
\ell\left(p_{k}(a, b, c, d ; \cdot) p_{l}(a, b, c, d ; \cdot)\right)=\frac{C}{\Gamma(b+c) \Gamma(b+d) \Gamma(c+d)} \sigma_{k}(a, b, c, d) \delta_{k, l},
$$

әде $\sigma_{n}(a, b, c, d)$ - выражсене, стоящее в правой части (3.2).

Иньми словами, многочлены Вильсона образуют ортогональную систему относительно скалярного произведения

$$
\langle f, g\rangle=\ell(f \bar{g})
$$

в пространстве многочленов.

ДоКАЗАТЕЛЬСТВо. Положим

$$
\ell(g)=\int_{0}^{\infty} g(s) w(s) d s
$$

где $w(s)$ - вес Вильсона. Тог да (3.7) выполнено и наше высказывание превращается в переформулировку соотношений ортогональности Вильсона.

Теперь полагаем

и

$$
\ell(g)=\int_{0}^{\infty} g(s) w_{1}(s) d s
$$

Получаем

$$
h_{k}(s)=(p+i s)_{k}(p-i s)_{k}
$$

$$
\begin{aligned}
\ell\left(h_{k}\right)= & \frac{\Gamma(p+v+k) \Gamma(p+u+k) \Gamma(u+v) \Gamma(q-u-v-p-k)}{\Gamma(q-p-k) \Gamma(q-u) \Gamma(p-v)} \\
= & \frac{\Gamma(u+v) \sin \pi(p-q)}{\Gamma(u-q) \Gamma(q-v) \sin \pi(u+v+p-q)} \\
& \times \frac{\Gamma(p+v+k) \Gamma(p+u+k) \Gamma(1-q+p+k)}{\Gamma(1-q+u+v+p+k)} .
\end{aligned}
$$

Итак, мы получаем, что система многочленов Вильсона

$$
p_{n}\left(p, u, v, 1-q ; s^{2}\right), \quad 4 n<q-p-u-v-1,
$$

ортогональна по весу (3.6). 
3.4. Соотношения ортогональности, связанные с формулой Дуголла. Рассмотрим вес

$$
w_{2}(t)=\sum_{-\infty}^{\infty} \frac{\alpha+t}{\prod_{j=1}^{4} \Gamma\left(a_{j}+\alpha+t\right) \Gamma\left(a_{j}-\alpha-t\right)} \delta(t-n),
$$

где $\delta(s-n)$ - дельта-функция, сосредоточенная в точке $n$. Для единообразия подставим $t=i s-\alpha$ и преобразуем это выражение к виду

$$
w_{2}(s):=\prod_{j=1}^{4} \frac{\sin \left(\pi\left(a_{j}+\alpha\right)\right)}{\pi}(i s) \sum_{-\infty}^{\infty}\left(\prod_{j=1}^{4} \Gamma\left(1-a_{j}-i s\right) \Gamma\left(1-a_{j}+i s\right) \delta(i s-\alpha-n)\right) .
$$

Чтобы применить лемму 3.2 , положим

$$
\begin{gathered}
\ell(g)=\int g(s) w_{2}(s) d s, \\
h_{k}(s)=\left(1-a_{1}+i s\right)_{k}\left(1-a_{1}-i s\right)_{k} .
\end{gathered}
$$

Тогда

$$
\begin{aligned}
\ell\left(h_{k}\right)= & \frac{\sin (2 \pi \alpha)}{2 \pi \Gamma\left(a_{2}+a_{3}\right) \Gamma\left(a_{2}+a_{4}\right) \Gamma\left(a_{3}+a_{4}\right)} \\
& \times \frac{\Gamma\left(a_{1}+a_{2}+a_{3}+a_{4}-k-3\right)}{\Gamma\left(a_{1}+a_{2}-k-1\right) \Gamma\left(a_{1}+a_{3}-k-1\right) \Gamma\left(a_{1}+a_{4}-k-1\right)} \\
= & \frac{\sin (2 \pi \alpha) \sin \pi\left(a_{1}+a_{2}\right) \sin \pi\left(a_{1}+a_{3}\right) \sin \pi\left(a_{1}+a_{4}\right)}{2 \pi \Gamma\left(a_{2}+a_{3}\right) \Gamma\left(a_{2}+a_{4}\right) \Gamma\left(a_{3}+a_{4}\right) \sin \pi\left(a_{1}+a_{2}+a_{3}+a_{4}\right)} \\
& \times \frac{\Gamma\left(-a_{1}-a_{2}+k+2\right) \Gamma\left(-a_{1}-a_{3}+k+2\right) \Gamma\left(-a_{1}-a_{4}+k+2\right)}{\Gamma\left(4-a_{1}-a_{2}-a_{3}-a_{4}+k\right)} .
\end{aligned}
$$

В итоге мы получаем, что система многочленов Вильсона

$$
p_{n}\left(1-a_{1}, 1-a_{2}, 1-a_{3}, 1-a_{4} ; s^{2}\right), \quad 4 n<a_{1}+a_{2}+a_{3}+a_{4}-3,
$$

ортогональна по весу Дуггола (3.8).

3.5. Соотношения ортогональности, связанные с интегралом Аски (0.5). Теперь рассмотрим вес Аски, т.е. вес, задаваемый подынтегральным выражением в $(0.5)$

$$
\frac{\Gamma(1-2 s) \Gamma(1+2 s)}{\prod_{j=1}^{4} \Gamma\left(a_{j}+s\right) \Gamma\left(a_{j}-s\right)} .
$$

Далее в обозначениях леммы 3.2 берем

Тогда

$$
\begin{gathered}
\ell(g)=\int_{-\infty}^{\infty} g(s) w_{3}(s) d s, \\
h_{k}(s)=\left(1-a_{1}+s\right)_{k}\left(1-a_{1}-s\right)_{k} .
\end{gathered}
$$

$$
\begin{aligned}
\ell\left(h_{k}\right)= & \frac{1}{\Gamma\left(a_{2}+a_{3}\right) \Gamma\left(a_{2}+a_{4}\right) \Gamma\left(a_{3}+a_{4}\right)} \\
& \times \frac{\Gamma\left(a_{1}+a_{2}+a_{3}+a_{4}-k-3\right)}{\Gamma\left(a_{1}+a_{2}-k-1\right) \Gamma\left(a_{1}+a_{3}-k-1\right) \Gamma\left(a_{1}+a_{4}-k-1\right)}
\end{aligned}
$$

и мы получили выражение, которое совпадает с (3.9) с точностью до постоянного множителя. Поэтому мы получили ту же систему ортогональных многочленов (3.10). 


\section{§4. Примеры индексных интегралов}

Сами по себе индексные интегралы - предмет достаточно известный (см., например, [18]). В этом параграфе мы приводим несколько забавных индексных интегралов, расширяюших интегралы $(0.1),(0.2),(2.2)-(2.5)$.

4.1. Индексное преобразование функции $(1+x)^{b-a}(x+y+1)^{-c-b}$ было вычислено в лемме 1.1. Применяя формулу обращения, получаем

$$
\begin{aligned}
& \int_{0}^{\infty}\left|\frac{\Gamma(a+i s) \Gamma(b+i s) \Gamma(c+i s)}{\Gamma(2 i s)}\right|_{2}^{2} F_{1}\left[\begin{array}{c}
c+i s, c-i s \\
a+c
\end{array} ;-y\right] \\
& \times{ }_{2} F_{1}\left[\begin{array}{c}
b+i s, b-i s \\
a+b
\end{array} ;-x\right] d s=\frac{\pi \Gamma(a+b) \Gamma(a+c) \Gamma(b+c)}{(1+x+y)^{c+b}} .
\end{aligned}
$$

4.2. Вьписывая формулу Планшереля для функций $(1+x)^{b-a}(x+y+1)^{-c-b}$ и $(1+x)^{-a-d}$, получаем (см. [4; терема 13$\left.]\right)$

$$
\begin{aligned}
& \int_{0}^{\infty}\left|\frac{\Gamma(a+i s) \Gamma(b+i s) \Gamma(c+i s) \Gamma(d+i s)}{\Gamma(2 i s)}\right|_{2}^{2} F_{1}\left[\begin{array}{c}
c+i s, c-i s \\
a+c
\end{array} ;-y\right] d s \\
& \quad=\frac{\pi \Gamma(a+b) \Gamma(a+c) \Gamma(a+d) \Gamma(b+c) \Gamma(b+d) \Gamma(c+d)}{\Gamma(a+b+c+d)}{ }_{2} F_{1}\left[\begin{array}{c}
b+c, c+d \\
a+b+c+d
\end{array} ;-y\right] .
\end{aligned}
$$

Эта формула совпадает (с точностью до перестановки букв) с формулой обращения для ${ }_{2} F_{1}\left[\begin{array}{c}a+c, a+d \\ a+b+c+d\end{array} ;-x\right]$ (см. лемму 1.2.c)). Отсюда вытекает вторая лемма Барнса (0.3) (это, конечно, не самое простое ее доказательство).

4.3. Применяя формулу Планшереля к паре функций $(1+x)^{b-a}(1+x+y)^{-b-c}$ и $(1+x)^{b-a}(1+x+z)^{-b-d}$, получаем

$$
\begin{aligned}
\frac{1}{\pi} \int_{0}^{\infty} & \left|\frac{\Gamma(a+i s) \Gamma(b+i s) \Gamma(c+i s) \Gamma(d+i s)}{\Gamma(2 i s)}\right|^{2} \\
& \times{ }_{2} F_{1}\left[\begin{array}{c}
c-i s, c+i s \\
a+c
\end{array} ;-y\right]{ }_{2} F_{1}\left[\begin{array}{c}
d-i s, d+i s ;-z \\
a+d
\end{array}\right] d s \\
= & \Gamma(c+a) \Gamma(c+b) \Gamma(d+a) \Gamma(d+b) \int_{0}^{\infty} \frac{x^{a+b-1}(1+x)^{b-a} d x}{(x+y+1)^{b+c}(x+z+1)^{b+d}} .
\end{aligned}
$$

В правой части стоит одно из интегральных представлений функции Аппеля $F_{1}$. Подставляя $z=y$, получаем

$$
\begin{aligned}
& \frac{1}{\pi} \int_{0}^{\infty}\left|\frac{\Gamma(a+i s) \Gamma(b+i s) \Gamma(c+i s) \Gamma(d+i s)}{\Gamma(2 i s)}\right|^{2} \\
& \quad \times{ }_{2} F_{1}\left[\begin{array}{c}
c-i s, c+i s \\
a+c
\end{array}-y\right]{ }_{2} F_{1}\left[\begin{array}{c}
d-i s, d+i s \\
a+d
\end{array} ;-y\right] d s \\
& =\frac{\pi \Gamma(a+b) \Gamma(a+c) \Gamma(a+d) \Gamma(b+c) \Gamma(b+d) \Gamma(c+d)}{\Gamma(a+b+c+d)} \\
& \quad \times{ }_{2} F_{1}\left[\begin{array}{c}
2 b+c+d, c+d \\
a+b+c+d
\end{array} ;-y\right] .
\end{aligned}
$$


4.4. Применим формулу Планшереля к паре функций $(1+x)^{b-a}(1+x+y)^{-e-b}$

$$
\begin{aligned}
& \text { и }{ }_{2} F_{1}\left[\begin{array}{c}
a+c, a+d \\
a+b+c+d
\end{array} ;-x\right] \text {. Получим } \\
& \frac{1}{\pi} \int_{0}^{\infty}\left|\frac{\Gamma(a+i s) \Gamma(b+i s) \Gamma(c+i s) \Gamma(d+i s) \Gamma(e+i s)}{\Gamma(2 i s)}\right|_{2}^{2} F_{1}\left[\begin{array}{c}
a+i s, a-i s \\
a+c
\end{array}-y\right] d s \\
& =\frac{1}{2 \pi i}(1+y)^{a-e} \Gamma(b+c) \Gamma(b+d) \Gamma(c+d) \\
& \quad \times \int_{-i \infty}^{i \infty} \Gamma\left[\begin{array}{c}
a+b+s, a+c+s, a+d+s, e-a-s,-s \\
a+b+c+d+s
\end{array}\right](1+y)^{s} d s
\end{aligned}
$$

(это сумма двух функций ${ }_{3} F_{2}$ ).

4.5. Этот список легко продолжить, даже наши леммы 1.1-1.2 использованы не полностью.

\section{Список литературы}

1. Wilson J. A. Somehypergeometric orthogonal polynomials // SIAM J. Math. Anal. 1980. V. 11. № 4. P. $690-701$.

2. Andrews G.E., Askey R., Roy R. Special functions. Cambridge: Cambridge University Press, 1999.

3. Koekoek R., Swarttouw R.F. Askey scheme of hypergeometric orthogonal polynomials and their $q$-analogues. Delft University of Technology, 1994; http://aw.twi.tudelft.nl/ $\sim$ koekoek/askey.html.

4. De Branges L. Tensor product spaces // J. Math. Anal. Appl. 1972. V. 38. P. 109-148.

5. Askey $R$. Beta integrals and the associated orthogonal polynomials // Number theory (Madras, 1987). Lecture Notes in Math. V. 1395. Berlin: Springer-Verlag, 1989. P. 84-121.

6. Rahman M., Suslov S. K. The Pearson equation and the beta integrals // SIAM J. Math. Anal. 1994. V. 25. № 2. P. 646-693.

7. Askey R., Wilson J. Some basic hypergeometric orthogonal polynomials that generalize Jacobi polynomials // Mem. Amer. Math. Soc. 1985. V. 54. №319.

8. Gustafson R. A. Some $q$-beta and Mellin-Barnes integrals on compact Lie groups and Lie algebras // Trans. Amer. Math. Soc. 1994. V. 341. № 1. P. 69-119.

9. Gustafson $R$. A. Some $q$-beta integrals on $\mathrm{SU}(n)$ and $\mathrm{Sp}(n)$ that generalize the Askey-Wilson and Nasrallah-Rahman integrals // SIAM J. Math. Anal. 1994. V. 25. № 2 . P. 441-449.

10. Romanovski V. I. Sur quelques classes nouwels of polynomes orthogonaux // Acad. Sci. Paris. 1929. V. 188. P. 1023-1025.

11. Askey R. An integral of Ramanujan and orthogonal polynomials // J. Indian Math. Soc. (N.S.). 1987. V. 51. P. 27-36.

12. Lesky P. A. Orthogonalitt von dualen Hahnpolynomen und continuous dualen Hahnpolynomen // Österreich. Akad. Wiss. Math.-Natur. Kl. Sitzungsber. II. 1996. V. 204. P. 81-95.

13. Lesky P. A. Endliche und unendliche Systeme von kontinuierlichen klassischen Orthogonalpolynomen // ZAMM Z. Angew. Math. Mech. 1996. V. 76. № 3. P. 181-184.

14. Lesky P. A. Unendliche und endliche Orthogonalsysteme von continuous Hahnpolynomen // Results Math. 1997. V. 31. № 1-2. P. 127-135.

15. Lesky P. A. Einordnung der Polynome von Romanovski-Bessel in das Askey-Tableau // ZAMM Z. Angew. Math. Mech. 1998. V. 78. №9. P. 646-648.

16. Peetre J. Correspondence principle for the quantized annulus, Romanovski polynomials, and Morse potential // J. Funct. Anal. 1993. V. 117. № 2. P. 377-400.

17. Borodin A., Olshanski G.I. Harmonic analysis on the infinite-dimensional unitary group and determinantal point processes // Preprint, http://arxiv.math.RT/0109194.

18. Slater L. J. Generalized hypergeometric functions. Cambridge: Cambridge University Press, 1966.

19. Marichev O. I. Handbook of integral transforms of higher transcedental functions. Chichester / New York: Ellis Horwood / John Willey, 1983. 
20. Прудников А.П., Брычков Ю.А., Маричев О. И. Интегралы и ряды. Дополнительные главы. Т. 3. М.: Наука, 1986.

21. Flensted-Jensen M., Koornwinder T. The convolution structure for Jacobi function expansions // Ark. Mat. 1973. V. 11. P. 245-262.

22. Koornwinder T. H. Jacobi functions and analysis on noncompact semisimple Lie groups // Special functions: group theoretical aspects and applications / ed. Askey R., Koornwinder T., Schempp W.. Dordrecht: Reidel, 1984. P. 1-85.

23. Koornwinder T. H. Special orthogonal polynomial systems mapped onto each other by the Fourier-Jacobi transform. Orthogonal polynomials and applications (Bar-le-Duc, 1984) // Lecture Notes in Math. V. 1171. Berlin: Springer-Verlag, 1985. P. 174-183.

24. Koornwinder T. H. Group theoretic interpretations of Askey's scheme of hypergeometric orthogonal polynomials. Orthogonal polynomials and their applications (Segovia, 1986) // Lecture Notes in Math. V. 1329. Berlin: Springer, 1988. P. 46-72.

25. Yakubovich S. B. Index transforms. London: World Scientific, 1996.

26. Неретин Ю. А. Индексное гипергеометрическое преобразование и имитация анализа ядер Березина на гиперболических пространствах // Матем. сб. 2001. Т. 192. №3. C. 83-114.

27. Бейтмен Г., Эрдейи А. Высшие трансцендентные функции. І: Гипергеометрическая функция. Функция Лежандра. М.: Наука, 1973.

28. Градитейн И. С., Рыжик И.М. Таблицы интегралов, сумм и произведений. М.: Физматгиз, 1963.

29. Rahman $M$. An integral representation of a ${ }_{10} \Phi_{9}$ and continuous bi-orthogonal $10 \Phi_{9}$ rational functions // Canad. J. Math. 1986. V. 38. P. 605-618.

30. Гаспер Г., Рахман М. Базисные гипергеометрические ряды. М.: Мир, 1993.

31. Bailey W. N. Generalized hypergeometric series. New York: Stechert-Hafner, 1964.

32. Nassrallah B., Rahman $M$. Projection formulas, a reproducing kernel and a generating function for $q$-Wilson polynomials // SIAM J. Math. Anal. 1985. V. 16. № 1. P. 186-197.

33. Nassrallah B., Rahman $M$. A $q$-analogue of Appell's $F_{1}$ function and some quadratic transformation formulas for nonterminating basic hypergeometric series // Rocky Mountain J. Math. 1986. V. 16. P. 63-82.

34. Бейтмен Г., Эрдейи А. Высшие трансцендентные функции. II: Функции Бесселя, функции параболического цилиндра, ортогональные многочлены. М.: Наука, 1974.

Институт теоретической и экспериментальной физики,

Поступила в редакцию Москва

20.11 .2001

E-mail: neretin@main.mccme.rssi.ru 